\title{
Acoustic and Electroglottographic Voice Characteristics in Chronic Cough and Paradoxical Vocal Fold Movement
}

\author{
Anne E. Vertigan ${ }^{a, d}$ Deborah G. Theodoros ${ }^{d}$ Alison L. Winkworth ${ }^{c}$ \\ Peter G. Gibson ${ }^{\text {b }}$ \\ ${ }^{a}$ Speech Pathology Department and bepartment of Respiratory and Sleep Medicine, Hunter Medical Research

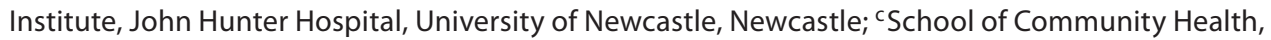 \\ Charles Sturt University, Albury; ${ }^{\mathrm{d}}$ Division of Speech Pathology, University of Queensland, Brisbane, Australia
}

\section{Key Words \\ Electroglottograph • Acoustic analysis • Chronic cough • Paradoxical vocal fold movement $\cdot$ Vocal cord dysfunction • Voice assessment $\cdot$ Muscle tension dysphonia $\cdot$ Voice disorders}

\begin{abstract}
Objective: Chronic cough (CC) and paradoxical vocal fold movement (PVFM) may be associated with voice problems. Objective acoustic and electroglottographic (EGG) measures have the capacity to delineate these vocal characteristics. This study investigated acoustic and EGG voice features of CC and PVFM. Patients and Methods: Acoustic and EGG findings were compared among 5 groups of participants. The first 3 groups, CC $(n=56)$, PVFM $(n=8)$ and combined CC-PVFM ( $n=55)$, included individuals with cough and respiratory symptoms that persisted despite medical treatment. Groups 4 and 5 included individuals with muscle tension dysphonia $(n=25)$ and healthy controls $(n=27)$. Results:
\end{abstract}

Institute where the research was conducted: John Hunter Hospital. (c) 2008 S. Karger AG, Basel

$1021-7762 / 08 / 0604-0210 \$ 24.50 / 0$

Fax +41613061234

E-Mail karger@karger.ch

www.karger.com
Accessible online at:

www.karger.com/fpl
Participants with CC/PVFM recorded reduced phonation times $(p<0.001)$, greater jitter $(p<0.001)$, reduced harmonic to noise ratio $(p=0.001)$, reduced phonation range $(p=$ 0.007 ) and shorter closed phase of vocal fold vibration ( $p=$ 0.006 ) in comparison to healthy controls. Females with CC had reduced fundamental frequency in connected speech $(p=0.009)$. There was consistent overlap between the participants with CC and those with PVFM. Duration of closed phase and fundamental frequency were lower in the participants with CC and PVFM than in those with muscle tension dysphonia. Conclusion: These results confirm abnormalities in acoustic and EGG voice features in CC and PVFM.

Copyright $\odot 2008$ S. Karger AG, Basel

\section{Introduction}

Chronic cough (CC) is defined as a cough persisting for longer than 8 weeks [1]. Once smoking and lung pathology have been excluded, the main aetiologies of CC are asthma, postnasal drip syndrome (PNDS), gastrooesophageal reflux (GER) and use of medications such as angiotensin-converting enzyme (ACE) inhibitors. CC can persist despite medical treatment [2]. Paradoxical vo- 
cal fold movement (PVFM) is a related condition whereby the vocal folds adduct episodically and involuntarily during inspiration. This phenomenon leads to reduced inspiratory airflow associated with signs of stridor and a perception of breathing discomfort characterized by the inability to inspire sufficient air [3]. Extrathoracic airway hyperresponsiveness is thought to be the primary underlying pathophysiology of PVFM [4]. PVFM has been associated with aetiological factors such as psychological conditions, laryngeal dystonia and airway irritation due to GER or sinusitis [5].

Voice problems have been noted to occur in persons with CC and PVFM and have significant quality of life implications [6, 7]. Recent studies have also identified an increased prevalence of self-reported voice symptoms, atypical findings during formal perceptual voice analysis, and endoscopic and stroboscopic abnormalities during phonation in individuals with CC and PVFM [8-10]. Speech pathologists are becoming increasingly involved in the management of CC and PVFM [11-13], however there is limited information to guide the management of voice problems occurring in these conditions. To date, there is a paucity of information in the literature regarding acoustic and electroglottographic (EGG) voice assessment in CC and PVFM, with no systematic comparison of these measures between the 2 conditions. Previous studies with small sample sizes have found increased jitter, decreased maximum phonation time (MPT) abnormalities in the soft phonation index, and abnormal variation in fundamental frequency in CC and PVFM [14, 15].

There are similarities in signs and symptoms occurring between CC, PVFM and voice disorders such as muscle tension dysphonia (MTD). These include throat clearing, voice difficulties $[9,16]$, an increased prevalence of extrathoracic airway hyperresponsiveness [17] and certain perceptual voice characteristics [10]. Despite this evidence, no large-scale studies of the acoustic and EGG features of phonation in CC and PVFM have been reported. It is unclear whether the similarities in perceptual voice measures between CC, PVFM and MTD are due to similar underlying mechanisms or whether they are a manifestation of the specificity of perceptual analysis.

In order to expand the current limited evidence on vocal function in CC and PVFM a prospective study was designed to (a) identify the sustained vowel acoustic and connected speech EGG voice features of persons with CC and PVFM; (b) determine the degree of overlap between the 2 conditions, and (c) compare acoustic and EGG voice features of persons with CC and PVFM with a group of individuals with MTD and with a group of healthy control speakers. It was hypothesized that there would be abnormalities in the vocal characteristics of persons with CC and PVFM and an overlap among CC, PVFM and MTD participant groups.

\section{Method}

The study involved a comparison of acoustic and EGG measures among 5 participant groups (table 1). The participants in groups 1, 2 and 3 were patients with symptoms of CC, PVFM and combined CC-PVFM, respectively. CC was defined as a cough that persisted for at least 2 months following medical treatment based on the Anatomic Diagnostic Protocol [22] including treatment for asthma, PNDS and GER, as well as withdrawal of ACE inhibitor medication (if used), and of sufficient severity to seek medical attention. Medical treatment had been effective for the primary condition(s) as determined by a respiratory physician. PVFM was defined as reduced inspiratory airflow due to involuntary and episodic glottal constriction associated with noisy inspiration and the perception of being unable to inspire sufficient air. This diagnosis was confirmed by a reduction of inspiratory flow on hypertonic saline challenge, where a reduction of $20 \%$ or more has been correlated with glottal constriction [18-20, 23]. The duration of CC and PVFM ranged from 4 months to 20 years. Inclusion criteria for these groups were persistence of symptoms for at least 2 months following medical treatment. Exclusion criteria included untreated asthma, allergy, GER or PNDS; current upper respiratory tract infection; abnormality on chest radiograph, chronic obstructive pulmonary disease, neurological voice disorder or dystonia. The participants in group 4 included 25 individuals diagnosed as having MTD by an otolaryngologist following flexible fiberoptic nasendoscopy. Inclusion criteria for this group were glottic or supraglottic constriction, with or without posterior gap or bowing, and a deviation in perceptual voice quality. Exclusion criteria included mass lesion, vocal fold paralysis and conversion dysphonia. The participants in group 5 served as healthy controls and had no history of voice or respiratory problems. None of the participants had received previous speech pathology intervention for CC, PVFM or dysphonia. There was no significant difference in gender distribution between the groups $\left(\chi^{2}=4.864\right.$, d.f. $\left.=4, \mathrm{p}=0.302\right)$.

Assessment of each participant involved acoustic analysis of sustained vowels and phonation range (PR), and an EGG analysis of connected speech. Acoustic assessment of sustained vowels utilized the PRAAT acoustics analysis program 3.9.27 [24] with signal input from a Studio Condenser Microphone NT3 (RODE) positioned $10 \mathrm{~cm}$ from the mouth and recorded directly into a personal computer with a sampling rate of $44.1 \mathrm{kHz}$. The participants were instructed to take a deep breath and say /a/ for as long as they possibly could at a comfortable pitch and loudness level [25, 26]. This task was performed 3 times following demonstration and practice. The participants were observed not to expire at the beginning or end of phonation and fundamental frequency was measured on each attempt. The task was repeated if vocal pitch was observed by the assessing speech pathologist to deviate mark- 
Table 1. Participant demographics, clinical presentation and medical history

\begin{tabular}{llllll}
\hline & $\begin{array}{l}\text { Combined } \\
\text { CC-PVFM }\end{array}$ & PVFM & CC & MTD & $\begin{array}{l}\text { Healthy } \\
\text { controls }\end{array}$ \\
\hline Participants & 55 & 8 & 56 & 25 & 27 \\
Female gender, \% & 76 & 63 & 75 & 92 & 70 \\
Mean age \pm SD, years & $55 \pm 14$ & $46 \pm 16$ & $59 \pm 13$ & $57 \pm 16$ & $37 \pm 16$ \\
Age range, years & $23-84$ & $20-65$ & $31-83$ & $20-80$ & $19-68$ \\
CC & + & - & + & N/A & - \\
PVFM & + & + & - & N/A & - \\
MTD & N/A & N/A & N/A & + & - \\
Inspiratory flow reduction, \% & $\geq 20$ & $\geq 20$ & $<20$ & N/A & N/A \\
& & & & & \\
Asthma, \% & 20 & 63 & 16 & 16 & 0 \\
GER, \% & 57 & 25 & 54 & 17 & 19 \\
PNDS, \% & 47 & 50 & 48 & 32 & 7 \\
Allergies, \% & 38 & 25 & 32 & 32 & 11 \\
ACE inhibitor, \% & 7 & 12 & 12 & 4 & $*$
\end{tabular}

Inspiratory flow: following hypertonic saline challenge. A reduction of $\geq 20 \%$ during hypertonic saline challenge has been correlated with glottal constriction [18-21]. Previous asthma treatment included inhaled corticosteroid and long-acting bronchodilator. GER: previous reflux treatment proton pump inhibitors. Previous PNDS treatment included topical nasal steroids and ingested antihistamines.

edly from modal speaking level or if phonation was interrupted by coughing. PR was calculated using a scale task whereby the participants vocalized an ascending scale on the phoneme /i/. This scale was recorded onto PRAAT and the number of semitones calculated. EGG analysis of a 2-minute sample of connected speech during a reading task using the extended Rainbow Passage [27] was conducted with the Laryngograph ${ }^{\circledR}$ Speech Studio (LSS), Laryngograph Ltd, UK [28, 29].

Five acoustic measures were calculated for each sustained vowel: MPT, mean fundamental frequency (AvFo), standard deviation of fundamental frequency (SDFo), jitter (percent) and harmonic to noise ratio (HNR). The steady-state vowel portion (minimum of $3 \mathrm{~s}$ ) as judged from the fundamental frequency trace was chosen for analysis. The average value of each measure across the 3 samples was calculated for each participant. The data analyzed from LSS included the modal fundamental frequency (DFx) measured in hertz, intensity measured in decibel SPL 3\% quarter tone intervals, irregularity (percent) of fundamental frequency (CFx) and the duration of the closed phase (percent) in each cycle of vocal fold vibration (Qx). The DFx was analyzed according to the first (DFx1) and second (DFx2) order distribution of fundamental frequency for each participant [30, 31]. Similarly, Qx was analyzed according to first (Qx1) and second $(\mathrm{Q} x 2)$ order distribution of the closed phase. The regularity and duration of $\mathrm{Qx}$ from vocal fold cycle to cycle is an important aspect of voice quality [31]. The CFx is based on the use of $6 \%$ pitch bins, provides an objective correlate of voice quality and is analogous to jitter in connected speech. A higher CFx value indicates less regular vocal fold vibration and correlates perceptually with poorer voice quality.

\section{Results}

A series of 2-way ANOVAs with covariate analysis to adjust the main group effect for age, followed by planned comparisons using independent $t$ tests, were used to analyze the results. Acoustic and EGG results are reported in table 2. A 2-way ANOVA revealed that MPT values demonstrated a significant main effect for group $(F=5.624$, $\mathrm{p}<0.001)$ and gender $(\mathrm{F}=10.107, \mathrm{p}=0.002)$ but no interaction effect between gender and participant group $(\mathrm{F}=$ $0.934, p=0.446$ ). Males had significantly longer MPT values than females. Independent $t$ tests revealed that the 3 CC/PVFM groups (combined CC-PVFM, PVFM and CC) had significantly shorter MPT values than the healthy controls $(t=4.219, \mathrm{p}<0.001)$. However, these values were not significantly different from the MTD group $(t=-0.756, p=0.455)$. There was no significant difference between the combined CC-PVFM and PVFM groups $(\mathrm{t}=$ $0.568, \mathrm{p}=0.571)$, the PVFM and CC groups $(\mathrm{t}=-0.316$, $\mathrm{p}=0.753)$ nor the combined CC-PVFM and CC groups $(\mathrm{t}=0.504, \mathrm{p}=0.615)$.

There was no significant difference in AvFo in sustained vowels among the participant groups $(\mathrm{F}=1.958$, $p=0.103$ ). Females had higher AvFo values than males $(\mathrm{F}=46.364, \mathrm{p}<0.001)$, but there was no interaction effect between gender and participant group $(\mathrm{F}=0.533, \mathrm{p}=$ 
Table 2. Acoustic and EGG results for participants in the combined CC-PVFM, PVFM, CC, MTD and healthy control groups

\begin{tabular}{lccccc}
\hline & $\begin{array}{c}\text { Combined } \\
\text { CC-PVFM }\end{array}$ & PVFM & CC & MTD & $\begin{array}{l}\text { Healthy } \\
\text { controls }\end{array}$ \\
\hline MPT, s & & & & $16.5 \pm 6.1$ \\
AvFo, Hz & $9.8 \pm 5.9$ & $11.2 \pm 6.7$ & $10.4 \pm 7.1$ & $10.8 \pm 7.1$ & \\
$\quad$ & & & & \\
$\quad$ Males & $114.1 \pm 27.4$ & $131.2 \pm 9.7$ & $117.2 \pm 18.3$ & $135.6 \pm 17.8$ & $120.8 \pm 19.5$ \\
$\quad$ Females & $175.4 \pm 35.4$ & $195.8 \pm 43.5$ & $173.7 \pm 52.1$ & $191.7 \pm 43.3$ & $205.4 \pm 36.2$ \\
SDFo, Hz & $23.4 \pm 19.3$ & $40.0 \pm 24.4$ & $23.5 \pm 18.5$ & $29.3 \pm 29.2$ & $11.9 \pm 12.3$ \\
PR, ST & $18.6 \pm 7.1$ & $16.4 \pm 5.2$ & $17.0 \pm 8.5$ & $17.6 \pm 6.3$ & $22.9 \pm 9.1$ \\
Jitter, \% & $2.3 \pm 1.9$ & $2.1 \pm 0.9$ & $2.7 \pm 2.4$ & $4.0 \pm 4.2$ & $1.6 \pm 2.4$ \\
HNR, dB & $18.2 \pm 5.3$ & $15.6 \pm 7.2$ & $16.8 \pm 6.7$ & $16.8 \pm 8.7$ & $21.5 \pm 3.8$ \\
DFx1, Hz & & & & & \\
$\quad$ Males & $106.3 \pm 22.0$ & $108.0 \pm 13.9$ & $103.7 \pm 8.5$ & $125.7 \pm 33.0$ & $105.3 \pm 15.1$ \\
$\quad$ Females & $174.9 \pm 23.4$ & $177.7 \pm 17.2$ & $165.4 \pm 31.8$ & $188.8 \pm 25.9$ & $187.8 \pm 27.3$ \\
DFx2, Hz & & & & & \\
$\quad$ Males & $107.0 \pm 21.3$ & $108.0 \pm 13.9$ & $103.7 \pm 8.5$ & $134.8 \pm 5.9$ & $105.3 \pm 15.1$ \\
$\quad$ Females & $172.2 \pm 26.8$ & $174.8 \pm 22.4$ & $165.2 \pm 31.6$ & $187.7 \pm 25.7$ & $188.2 \pm 28.0$ \\
CFx, \% & $16.6 \pm 12.9$ & $22.4 \pm 21.0$ & $20.2 \pm 14.3$ & $22.9 \pm 14.5$ & $15.2 \pm 7.4$ \\
Intensity, dB & $70.7 \pm 6.6$ & $73.2 \pm 8.6$ & $70.8 \pm 5.9$ & $71.5 \pm 5.3$ & $73.5 \pm 4.9$ \\
Qx1, \% & $39.4 \pm 9.9$ & $34.1 \pm 7.0$ & $37.5 \pm 10.8$ & $47.9 \pm 12.8$ & $43.8 \pm 11.7$ \\
Qx2, \% & $34.9 \pm 17.7$ & $33.8 \pm 18.4$ & $33.8 \pm 14.8$ & $46.6 \pm 21.7$ & $47.2 \pm 21.8$ \\
\hline
\end{tabular}

Values are means \pm SD. $S T=$ Semitones.

0.712). Due to the large variability in AvFo results the Welch test was used to provide corrected $\mathrm{F}$ tests. This analysis found no significant difference between the groups for either male $(\mathrm{F}=1.103, \mathrm{p}=0.435)$ or female $(\mathrm{F}=2.685, \mathrm{p}=0.055)$ participants.

The main effect for group was significantly different in SDFo analysis $(\mathrm{F}=4.231, \mathrm{p}=0.003)$. There was no main effect for gender $(\mathrm{F}=0.061, \mathrm{p}=0.806)$ and no interaction effect between gender and participant group $(\mathrm{F}=1.953$, $\mathrm{p}=0.104)$. The $3 \mathrm{CC} / \mathrm{PVFM}$ groups had significantly higher SDFo results than the healthy controls $(t=-4.647$, $\mathrm{p}<0.001)$ but were not significantly different from the MTD group $(t=0.096, p=0.924)$. There was no significant difference between the combined CC-PVFM and PVFM groups $(t=2.048, p=0.042)$, the PVFM and CC groups $(t=0.029, \mathrm{p}=0.977)$ or the combined CC-PVFM and CC groups $(\mathrm{t}=-2.036, \mathrm{p}=0.043)$.

$\mathrm{PR}$ values were significantly different between the groups $(\mathrm{F}=2.973, \mathrm{p}=0.021)$. There was no main effect for gender $(\mathrm{F}=3.317, \mathrm{p}=0.070)$ and no interaction effect between gender and participant group $(\mathrm{F}=1.441, \mathrm{p}=$ 0.223 ). The 3 CC/PVFM groups had significantly lower $\mathrm{PR}$ values than the healthy control group $(\mathrm{t}=2.850, \mathrm{p}=$ $0.007)$ but similar results to the MTD group $(t=0.130$,

Chronic Cough and Paradoxical Voca Fold Movement $p=0.897)$. There was no significant difference between the combined CC-PVFM and PVFM groups $(t=-0.709$, $\mathrm{p}=0.479)$, the combined CC-PVFM and CC groups $(\mathrm{t}=$ $-1.091, \mathrm{p}=0.277)$ or the PVFM and CC groups $(\mathrm{t}=0.189$, $\mathrm{p}=0.850)$.

Jitter results were significantly different between the participant groups $(\mathrm{F}=3.378, \mathrm{p}=0.001)$. There was no main effect for gender $(\mathrm{F}=0.955, \mathrm{p}=0.330)$ and no interaction effect between gender and participant group $(\mathrm{F}=$ $0.957, \mathrm{p}=0.433$ ). Jitter values were significantly higher in the $3 \mathrm{CC} / \mathrm{PVFM}$ groups than the healthy control group $(t=-3.865, p<0.001)$. There was no significant difference between the $3 \mathrm{CC} / \mathrm{PVFM}$ groups and the MTD group $(t=0.300, p=0.764)$, the combined CC-PVFM and PVFM groups $(t=1.161, p=0.247)$, the PVFM and CC groups $(t=-0.867, p=0.387)$ or the combined CC-PVFM and CC groups $(t=0.583, p=0.560)$.

There was a significant difference in $\mathrm{HNR}$ values among the participant groups $(\mathrm{F}=3.319, \mathrm{p}=0.012)$ but no significant gender difference $(\mathrm{F}=1.492, \mathrm{p}=0.224)$ and no interaction effect between gender and group $(\mathrm{F}=$ $0.686, p=0.603)$. The 3 CC/PVFM groups had significantly lower HNR values than healthy controls $(t=3.923$, $p=0.001$ ) but were not significantly different from the 
MTD group $(t=-0.013, p=0.989)$. There was no significant difference between the combined CC-PVFM and PVFM groups $(t=-1.103, p=0.271) p=0.805)$, the PVFM and CC groups $(t=-1.210, p=0.228)$ or the combined CC-PVFM and CC groups $(t=0.497, p=0.620)$.

Main effects for group were significant for DFx1 ( $F=$ $3.068, \mathrm{p}=0.018)$ and DFx2 $(\mathrm{F}=3.123, \mathrm{p}=0.017)$. Females had significantly higher DFx1 $(\mathrm{F}=198.762, \mathrm{p}<0.001)$ and DFx2 $(\mathrm{F}=173.042, \mathrm{p}<0.001)$ values than males. There was no interaction effect between gender and participant group for either DFx1 $(\mathrm{F}=0.605, \mathrm{p}=0.660)$ or $\mathrm{DFx} 2(\mathrm{~F}=0.753, \mathrm{p}=0.558)$. The $3 \mathrm{CC} / \mathrm{PVFM}$ groups had significantly lower DFx1 and DFX2 values than the MTD group $(t=3.261, p=0.001 ; t=3.823, p=0.001$, respectively) and females in the CC group had significantly lower values than the healthy controls $(p=0.009 ; p=0.001$ ). There was no significant difference in DFx1 and DFX2 values between the combined CC-PVFM and PVFM groups $(\mathrm{t}=-0.912, \mathrm{p}=0.397 ; \mathrm{t}=-0.925, \mathrm{p}=0.397)$, PVFM and CC groups $(\mathrm{t}=0.444, \mathrm{p}=0.672 ; \mathrm{t}=0.542, \mathrm{p}=0.589)$ or the combined CC-PVFM and CC groups $(\mathrm{t}=-1.048$, $\mathrm{p}=0.297 ; \mathrm{t}=-0.822, \mathrm{p}=0.413)$ on this measure.

There was no main effect for group $(\mathrm{F}=1.715, \mathrm{p}=$ $0.149)$ or gender $(\mathrm{F}=1.296, \mathrm{p}=0.257)$ in the analysis of $\mathrm{CFx}$. There was no interaction effect between gender and participant group $(\mathrm{F}=1.257, \mathrm{p}=0.289)$. Analysis of intensity results revealed no main effect for group ( $\mathrm{F}=$ $1.190, \mathrm{p}=0.355)$ or gender $(\mathrm{F}=0.177, \mathrm{p}=0.675)$. There was no interaction effect between gender and participant group $(\mathrm{F}=1.157, \mathrm{p}=0.332)$.

Qx1 and Qx2 values were significantly different between participant groups ( $\mathrm{Qx} 1: \mathrm{F}=3.346, \mathrm{p}=0.012$; $\mathrm{Qx} 2$ : $\mathrm{F}=3.806, \mathrm{p}=0.006$ ). There was a significant main effect for gender, with females demonstrating longer Qx1 ( $\mathrm{F}=$ $10.888, \mathrm{p}=0.001)$ and $\mathrm{Qx} 2(\mathrm{~F}=4.035, \mathrm{p}=0.046)$ values than males. There was no interaction effect between gender and participant group for either Qx1 $(\mathrm{F}=1.731, \mathrm{p}=$ $0.146)$ or $\mathrm{Qx} 2(\mathrm{~F}=2.213, \mathrm{p}=0.071)$. Planned contrasts revealed no significant difference in the $\mathrm{Qx} 1$ values of the $3 \mathrm{CC} / \mathrm{PVFM}$ groups compared to the healthy control group $(t=2.577, p=0.011)$ but lower $Q x 2$ values $(t=2.798$, $\mathrm{p}=0.006)$. $\mathrm{Qx} 1$ and $\mathrm{Qx} 2$ values in the $3 \mathrm{CC} / \mathrm{PVFM}$ groups were significantly lower than in the MTD group $(\mathrm{t}=$ 3.752, $\mathrm{p}=0.001 ; \mathrm{t}=2.343, \mathrm{p}=0.010)$. There was no significant difference in $\mathrm{Qx} 1$ or $\mathrm{Qx} 2$ values between the combined CC-PVFM and PVFM $(\mathrm{t}=-1.229, \mathrm{p}=0.221$; $\mathrm{t}=-0.129, \mathrm{p}=0.897)$, PVFM and CC $(\mathrm{t}=782, \mathrm{p}=0.435$; $\mathrm{t}=-0.001, \mathrm{p}=0.999)$ or between the combined CC-PVFM and CC groups for this measure $(t=-0.934, p=0.352$; $\mathrm{t}=-0.276, \mathrm{p}=0.783$ ).

\section{Discussion}

The current study identified several key acoustic and EGG vocal features in persons with CC and PVFM, including abnormalities in MPT, jitter, HNR, DFx, SDFo and $\mathrm{Qx}$, in comparison to healthy controls. There were no significant differences between the $3 \mathrm{CC} / \mathrm{PVFM}$ groups on any acoustic or EGG measure. The reduced Qx values suggest that people with CC and PVFM might have reduced vocal fold contact during phonation and a tendency toward vocal hypofunction which are consistent with previous studies that found abnormalities in the Soft Phonation Index due to incomplete vocal fold adduction [15]. The reduced MPT values identified in the study may be due to either glottal or respiratory insufficiency. In order to delineate this finding further it would be necessary to compare MPT results to baseline respiratory function in persons with CC/PVFM. Such data were not available in the current study, but previous work by our group has found that participants with CC/PVFM have adequate respiratory function to sustain speech. With respect to fundamental frequency, the reduced $D F x$ values in connected speech in female participants were consistent with an increased prevalence of a deeper voice identified through patient self-report in females with CC/PVFM [9]. It is unlikely that lowered DFx values were due to vocal fold oedema secondary to persisting GER as participants with untreated GER were excluded from the study. Reduced fundamental frequency may suggest reduced vocal fold tension caused by greater activation of the thyroarytenoid muscle and reduced activity in the cricothyroid muscle during phonation, and decreased subglottal pressure, and increased susceptibility to vocal abuse and hormonal influences [32]. Specific conclusions about endocrine factors are only speculative as menopausal status was not systematically determined in these individuals. Participants in the CC/PVFM groups demonstrated higher SDFo values than healthy controls, which is consistent with poor vocal stability and difficulty modulating pitch $[8,33]$. Acoustic correlates of vocal quality were affected in CC and PVFM. CFx is unique to the LSS and yet to be routinely reported in studies of voice disorders, CC and PVFM; however, normative data for the LSS have recently been published [34]. The large standard deviations in the results of the current study indicate that the severity of acoustic and physiological measures in people with CC and PVFM is variable. Although the younger age of the healthy controls may have influenced these results, the ANCOVA demonstrated no significant interaction between group and age for any of the measures in the study. 
The participants in the MTD group differed significantly from those in the 3 CC/PVFM groups in terms of Qx and DFx. Qx values were shorter in the CC/PVFM groups than in the MTD group, indicating that the latter group may have had a greater tendency towards hyperadduction of the vocal folds than the CC/PVFM groups. The results suggest that MTD is a discrete condition from $\mathrm{CC}$ and PVFM. The DFx values for the participants in the combined CC-PVFM and CC groups were significantly lower than for the participants in the MTD group. These findings suggest that individuals with CC and PVFM might use lower subglottic pressure or lower vocal fold tension than those with MTD. Despite the differences between the MTD group and the CC/PVFM groups in the current study there was considerable overlap in some acoustic voice and EGG measures between the groups. The similarities in vocal quality and duration measures were interesting given that the participants in the $3 \mathrm{CC} /$ PVFM groups had been referred to speech pathology for behavioural management of cough and respiratory signs and symptoms rather than for management of a voice disorder. It is unclear whether the similarity in results between the groups is a function of the inadequate discrimination of these measures themselves, evidence of overlapping diagnoses or a reflection of limited difference between the clinical groups.

The main clinical implication of this study is that despite variability in the acoustic and EGG measures of voice in participants with CC and PVFM these individuals were more likely to produce values in the abnormal range. The possibility of co-occurring voice problems in these populations needs to be considered. These findings add objective support to the hypothesis that CC can be associated with dysfunction at the laryngeal level. These findings may suggest that some other physiological abnormality such as increased cough reflex sensitivity is common to both CC groups. A further consideration is that many people with CC report that talking triggers the cough [9]. Reduced vocal fold contact during phonation in CC and PVFM could be due to attempts to reduce stimulation of pressure receptors in the larynx during phonation that trigger cough. It may be appropriate to optimize the efficiency of vocal fold vibratory patterns during phonation in order to reduce any unnecessary laryngeal irritation. Direct voice therapy techniques might be required in addition to management of the cough and respiratory signs and symptoms in CC and PVFM. In addition to retraining phonatory patterns, promoting training nasal breathing may decrease laryngeal irritation associated with cough. Previous research has reported oral breathing in a significant proportion of individuals with CC [9] and that oral breathing has been associated with increased phonation threshold pressure [35].

Future protocols may benefit from including more challenging tasks that are more sensitive to change (e.g. sustaining vowels at lowest intensity and highest frequency). Analysis of voice in larger groups with PVFM without cough is required in order to establish a more valid understanding of this condition. The small sample size of the PVFM group, while reflective of the prevalence of this condition, remains a limitation of this study.

\section{Conclusion}

Key features of CC and PVFM were identified in the current study and included reduced MPT, HNR, PR, DFx and Qx along with increased SDFo and jitter, compared to healthy controls. The lack of significant difference in acoustic and EGG voice measures among the $3 \mathrm{CC} /$ PVFM groups is consistent with previous studies of selfreported voice problems and perceptual voice analyses suggesting a common underlying mechanism in these 2 conditions. Finally, despite some similarities in the vocal characteristics between the $3 \mathrm{CC} / \mathrm{PVFM}$ groups and the MTD group, several vocal features of the CC/PVFM group were suggestive of hypofunction and provide objective support for problems at the laryngeal level in these conditions.

\section{Acknowledgements}

This study was supported by a grant from Jennifer Thomas through the Hunter Medical Research Institute. Prof. P. Gibson is an NHMRC Practitioner Fellow. Anne Vertigan holds a scholarship from the NHMRC Centre for Clinical Research Excellence in Respiratory and Sleep Medicine.

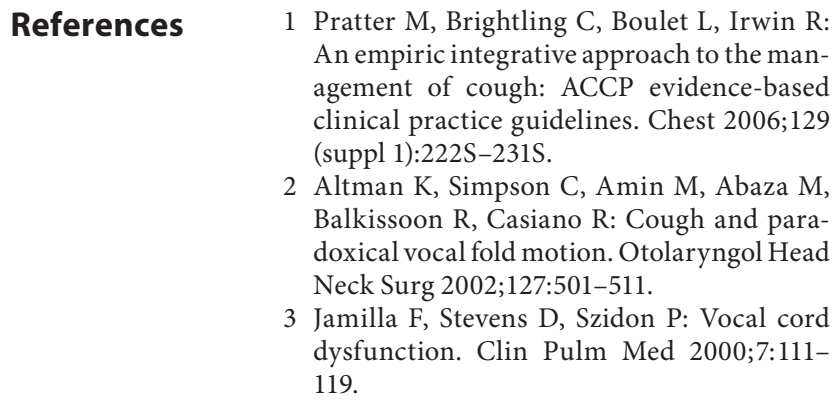

Folia Phoniatr Logop 2008;60:210-216 
4 Brugman S: What's this thing called vocal cord dysfunction? Chest Online; December 2006

5 Mathers-Schmidt BA: Paradoxical vocal fold motion: a tutorial on a complex disorder and the speech-language pathologist's role. Am J Speech Lang Pathol 2001;10:111-125.

6 French C, Irwin R, Curley F, Krikorian C: Impact of chronic cough on quality of life. Arch Intern Med 1998;158:1657-1661.

7 Andrianopoulos M, Gallivan G, Gallivan K: PVCM, PVCD, EPL, and irritable larynx syndrome: what are we talking about and how do we treat it? J Voice 2000;14:607-618.

8 Treole K, Trudeau M, Forrest L: Endoscopic and stroboscopic description of adults with paradoxical vocal fold dysfunction. J Voice 1999;13:143-152.

9 Vertigan AE, Theodoros DG, Gibson PG, Winkworth AL: Voice and upper airway symptoms in people with chronic cough and paradoxical vocal fold movement. J Voice 2007;21:361-383

10 Vertigan A, Theodoros D, Winkworth A, Gibson P: Perceptual voice characteristics in chronic cough and paradoxical vocal fold movement. Folia Phoniatr Logop 2007;59: 256-267.

- 11 Murry T, Tabaee A, Aviv J: Respiratory retraining of refractory cough and laryngopharyngeal reflux in patients with paradoxical vocal fold movement disorder. Laryngoscope 2004;114:1341-1345.

-12 Sullivan M, Heywood B, Beukelman D: A treatment for vocal cord dysfunction in female athletes: an outcome study. Laryngoscope 2001;111:1751-1755.

-13 Vertigan AE, Theodoros DG, Gibson PG, Winkworth AL: Efficacy of speech pathology management for chronic cough: a randomised, single blind, placebo controlled trial of treatment efficacy. Thorax 2006;61: 1065-1069.
4 Murry T: Chronic cough: in search of the etiology. Sem Speech Lang 1998;19:83-91.

15 Zelcer S, Henri C, Tewfik TL, Mazer B: Multidimensional voice program analysis (MDVP) and the diagnosis of paediatric vocal cord dysfunction. Ann Allergy Asthma Immunol 2002;88:601-608.

16 Morrison M, Rammage L, Emami AJ: The irritable larynx syndrome. J Voice 1999;13: 447-455.

17 Vertigan A, Gibson P, Theodoros D, Borgas T, Winkworth A, Reid C: Involuntary glottal closure during inspiration in muscle tension dysphonia. Laryngoscope 2006;116:643649.

18 Gibson P, Taramarcaz P, Borgas T: Evaluation of diagnostic tests for vocal cord dysfunction. Am J Respir Crit Care Med 2004; 169:A317.

19 Taramarcaz P, Grissell TV, Borgas T, Gibson P: Transient post-viral vocal cord dysfunction. J Allergy Clin Immunol 2004;114:14711472.

20 Ryan N, Gibson P: Cough reflex hypersensitivity and upper airway hyperresponsiveness in vocal cord dysfunction with chronic cough. Respirology 2006;11(suppl 2):A48

-21 Bucca C, Rolla G, Scappaticci E, Baldi S, Caria $\mathrm{E}$, Oliva A: Histamine hyperresponsiveness of the extrathoracic airway in patients with asthmatic symptoms. Allergy 1991;46: 147-153.

22 Irwin R, Boulet L, Cloutier M, Fuller R, Gold $\mathrm{P}$, Hoffstein V, et al: Managing cough as a defence mechanism and as a symptom: a consensus panel report of the American College of Chest Physicians. Chest 1998;114(2 Suppl Managing):133S-181S.

23 Bucca C, Rolla G, Brussino L, DeRose V, Bugiani M: Are asthma-like symptoms due to bronchial or extrathoracic airway dysfunction? Lancet 1995;346:791-795.
24 Boermsa P, Weenick D: Accurate short-term analysis of the fundamental frequency and the harmonic to noise ratio of a sampled sound. IFA Proc 1993;17:97-110.

25 Hirano M: Clinical Examination of Voice. Vienna, Springer, 1981.

26 Baken R: Clinical Measurement of Speech and Voice. Needham Heights, Allyn \& Bacon, 1987.

27 Fairbanks G: Voice and Articulation Drill Book, ed 2. New York, Harper \& Row, 1960.

28 Fourcin A: Laryngographic assessment of phonatory function. ASHA Rep 1981;11: 116-127.

29 Titze I, Horii Y, Scherer R: Some technical considerations in voice perturbation measurements. J Speech Hearing Res 1987;30: 252-260.

30 Abberton E: Aspects of voice quality in women. 2002. http://www.phon.ucl.ac. uk.home/evelyn/Korea02/abberton 0502 . doc (accessed March 17, 2005).

31 Fourcin A, McGlashan J, Blowes R: Measuring voice in the clinic: Laryngograph speech studio analysis. 6th Aust Voice Symp, Adelaide, 2002

32 McGarvey L: Idiopathic chronic cough: a real disease or a failure of diagnosis? Cough 2005; 1:9.

33 Gorham-Rowan M: Acoustic measures of vocal stability during different speech tasks in young women using oral contraceptives: a retrospective study. Eur J Contracept Reprod Health Care 2004;9:166-172.

34 Ptok M, Iven C, Jessen M, Schwemmle C: Objektiv gemessene Stimmlippenschwingungsirregularität vs. subjektiver Eindruck der Rauigkeit. HMO 2006;54:132-138.

35 Sivasanker M, Fisher K: Oral breathing increases Pth and vocal effort by superficial drying of the vocal fold mucosa. J Voice 2002;16:172-181. 